\title{
ESTUDO DA ISQUEMIA E REPERFUSÃO EM RETALHOS CUTÂNEOS DE RATOS ${ }^{1}$
}

\section{THE STUDY OF THE ISCHEMIA AND REPERFUSION IN SKIN FLAPS OF RATS}

\author{
Frederico Alonso Sabino de Freitas ${ }^{2}$ \\ Carlos Eli Piccinato ${ }^{3}$ \\ Antônio Dorival Campos ${ }^{4}$ \\ Jesualdo Cherri ${ }^{3}$
}

\section{RESUMO}

Introdução - Múltiplos fatores têm sido implicados na patogênese da lesão de isquemia/reperfusão da pele, incluindo as espécies reativas de oxigênio. Objetivo - Estudar a lesão de isquemia/reperfusão em retalhos cutâneos de ratos avaliando os níveis teciduais do malonildialdeído (MDA) e xantina oxidase (XO). Métodos - Foram utilizados 8 ratos Wistar, com peso entre $300-400 \mathrm{~g}$, sendo confeccionados 2 retalhos epigástricos por animal (controle e experimento), um deles submetido à $16 \mathrm{~h}$ de isquemia (RI) seguida de 45 min de reperfusão (RR) e o outro controle (RC). Foram colhidas 3 biópsias de pele dos retalhos (RC, RI, RR) e encaminhadas para dosagem de MDA e XO. Resultados - A análise bioquímica mostrou aumento significativo dos níveis teciduais de MDA e XO após a reperfusão em relação aos retalhos controles. Conclusão - Retalhos epigástricos de ratos submetidos à $16 \mathrm{~h}$ de isquemia e $45 \mathrm{~min}$ de reperfusão apresentam elevação dos níveis teciduais de MDA e XO, caracterizando a lipoperoxidação da membrana celular. Disponível em URL: http://www.scielo.br/acb

Descritores - Isquemia; Reperfusão; Retalho epigástrico; Lipoperoxidação; Ratos.

\section{ABSTRACT \\ Introduction - Multiple factors have been implicated in the pathogenesis of reperfusion injury in the skin, including the reactive oxygen species.}

Objective - The aim was to evaluate the effect of reperfusion injury in the rat skin flap evaluated by tissue assay for malonyldialdehyde (MDA) and xanthine oxidase (XO). Methods - 8 Wistar rats were used, between $300-400 \mathrm{~g}$ weight and two identical epigastric flaps were raised in each animal (control and experiment), the vasculature of one flap was left intact and in the second flap the arterial pedicle was clamped for 16 hours and reperfused for 45 minutes. Skin samples were obtained from each flap after these periods of time and submitted to MDA and XO analysis. Results - Reperfused flaps had significantly increased MDA and XO values compared to the control flaps biopsies. Conclusion - The lipid peroxidation levels were higher in the rat epigastric skin flaps subjected to 16 hours of ischemia and 45 minutes of reperfusion.

Key words - Ischemia; Reperfusion; Epigastric flap; Lipid peroxidation; Rats.

\section{INTRODUÇÃO}

As espécies reativas de oxigênio (ERO) têm sido implicadas numa variedade de processos patológicos, incluindo a lesão de isquemia/reperfusão promovendo a necrose de tecidos reperfundidos e retalhos microcirúrgicos ${ }^{1,2}$.

O mecanismo de lesão celular, na presença de ERO, parece estar ligado à lipoperoxidação da membrana celular. Este processo pode ser medido indiretamente pela dosagem de malonildialdeído (MDA), seu produto estável final ${ }^{3}$.

\footnotetext{
Trabalho realizado no Laboratório de Cirurgia Experimental do Departamento de Cirurgia e Anatomia - FMRP-USP

Mestre em Clínica Cirúrgica, Médico Assistente do HC-FMRP-USP

Professor Associado do Departamento de Cirurgia e Anatomia - FMRP-USP

Professor Associado do Departamento de Medicina Social - FMRP - USP
} 
Vários estudos relacionam a lesão de isquemia/ reperfusão à produção de ERO, entretanto existem controvérsias quanto aos mecanismos responsáveis pela gênese destas moléculas ${ }^{4}$.

Os dois sistemas enzimáticos mais estudados são os da xantina oxidase (XO) e da nicotinamida adenina dinucleotídeo oxidase (NADPH) ${ }^{5}$. Este último sistema está localizado na membrana celular dos neutrófilos que, quando ativados, também produzem radicais superóxidos. O primeiro faz parte do metabolismo intracelular'.

Está bem estabelecido que estes radicais são formados precocemente durante a reperfusão, normalmente na primeira hora ${ }^{7}$.

Vários autores estudaram retalhos cutâneos em ratos na tentativa de reproduzir, experimentalmente, as lesões cutâneas decorrentes da isquemia /reperfusão ${ }^{8,9}$.

O retalho epigástrico do rato apresenta um padrão axial com circulação cutânea bem definida sendo que a linha média delimita a transição para sua porção contralateral, nutrida pelo outro pedículo, baseado na artéria e veia epigástricas, ramos diretos dos vasos femorais contralaterais ${ }^{10}$.

Utilizando este retalho como modelo experimental, objetivou-se, no presente trabalho, estudar o fenômeno de isquemia e reperfusão em tecido cutâneo, através da dosagem tecidual de MDA e XO.

\section{MÉTODOS}

Foram utilizados 8 ratos da raça Wistar, sem distinção de sexo, com idade cronológica semelhante e peso entre 300-400g, provenientes do Biotério Central da Faculdade de Medicina de Ribeirão Preto da Universidade de São Paulo (FMRP-USP).

Os animais foram acomodados em gaiolas de aço inoxidável, respeitando-se critérios populacionais, e mantidos em sala fechada, com iluminação artificial controlada, temperatura aproximada de $22^{\circ} \mathrm{C}$, ração e água ad libitum.

Os experimentos foram realizados na sala de cirurgia do Laboratório de Técnica Cirúrgica e Cirurgia Experimental do Departamento de Cirurgia e Anatomia da FMRP- USP.

Foram operados 8 ratos, confeccionando-se 2 retalhos epigástricos por animal, sendo um retalho controle e o contralateral submetido à $16 \mathrm{~h}$ de isquemia e, posteriormente, a 45 min de reperfusão, totalizando 16 retalhos. Padronizou-se o retalho do hemi-abdome direito como sendo o controle.

Os retalhos cutâneos foram assim considerados: 8 retalhos controles (RC) e 8 retalhos isquêmicos (RI) e 8 reperfundidos (RR).
Biópsias cutâneas destes retalhos foram colhidas ao final de $16 \mathrm{~h}$ de isquemia e ao término do período de reperfusão. A biópsia de pele controle foi colhida do lado contralateral e todo material foi enviado para dosagem tissular de MDA e XO.

$\mathrm{O}$ modelo de isquemia e reperfusão de tecido cutâneo utilizado foi o retalho cutâneo epigástrico do rato (padrão axial) ${ }^{11}$.

$\mathrm{O}$ animal foi anestesiado com éter inalatório e posicionado em banqueta com as patas fixas. Fez-se a tricotomia de toda região abdominal e realizou-se uma incisão na linha média, desde o xifóide à região genital com extensão do rebordo costal (superiormente) e até a região inguinal (inferiormente) e dissecou-se o plano supramuscular, incluindo o panículo carnoso, com visualização direta dos ramos epigástricos. Neste ponto delimitou-se a extensão lateral do retalho, incluindo o ramo lateral do tronco epigástrico. O pedículo (base) desta forma mantém largura suficiente apenas para 0 tronco epigástrico superficial ("em ilha") e as dimensões da pele do retalho retangular mediram $4 \times 6 \mathrm{~cm}$. Com o auxílio do microscópio (D.F. Vasconcelos São Paulo, mod. FC 250, aumento 10 vezes), prosseguiu-se a dissecção profundamente na região inguinal com identificação dos vasos femorais. Sua porção caudal à saída do ramo epigástrico superficial e os ramos musculares foram cauterizados com eletrocautério bipolar, tornando o retalho nutrido apenas pelo pedículo femoral. Uma pinça microvascular $\left(\right.$ Acland- $\mathrm{V}_{1}$ ) foi colocada na artéria femoral, ocluindo-a, cranial à saída dos vasos epigástricos, provocando a isquemia do retalho. O mesmo procedimento foi realizado do lado contralateral, sem contudo provocar oclusão do pedículo vascular. Os retalhos foram devolvidos aos seus leitos simultaneamente e suturados com pontos contínuos de mononylon 5-0. Foram confeccionados colares cervicais com filmes de raios X e adaptados aos animais para evitar a autocanibalização e a seguir o animal foi reconduzido à gaiola com água e ração $a d$ libitum.

Após $16 \mathrm{~h}$ os ratos foram novamente anestesiados e foram colhidos dois fragmentos de pele $(1 \times 1 \mathrm{~cm})$ com bisturi lâmina 15 , um de cada retalho: retalho controle (RC) e retalho isquêmico (RI), em pontos distais, simétricos e equidistantes da base. O material foi enviado imediatamente ao laboratório e processado para dosagem de MDA e XO. Neste momento liberou-se a pinça vascular e o fluxo foi restabelecido. A patência da artéria foi observada sob visão direta da pulsação ao microscópio e sinais de permeabilidade ${ }^{12}$ (teste vazio/ cheio). Após 45 min de reperfusão, nova biópsia caudal do retalho reperfundido (RR) foi colhida e enviada 
para análise bioquímica de MDA e XO. A seguir procedeu-se à eutanásia, respeitando-se as regras do manual de tratamento de animais de experimentação.

As análises bioquímicas de MDA e XO foram realizadas no Laboratório de Cirurgia Experimental do Departamento de Cirurgia e Anatomia.

A dosagem do malonildialdeído foi realizada pelo método do tiobarbiturato ${ }^{13}$. A dosagem da atividade enzimática da $\mathrm{XO}$ foi realizada pela técnica modificada para tecido cutâneo ${ }^{14}$.
Os dados referentes às concentrações de MDA e $\mathrm{XO}$ em retalhos controles (RC), retalhos isquêmicos (RI) e reperfundidos (RR) foram analisados com base no teste não-paramétrico de Friedman, aproximado para mais de 2 amostras correlacionadas ${ }^{15}$. Considerou-se o nível de significância a 5\%.

\section{RESULTADOS}

As medianas das concentrações tissulares de MDA nos retalhos controle (RC), após $16 \mathrm{~h}$ de isquemia (RI) e 45 min de reperfusão (RR) estão expostas na tabela 1.

Tabela 1 - Mediana das concentrações tissulares de malonildialdeído- MDA em retalhos cutâneos controles $(\mathrm{RC})$, isquêmicos (RI) e reperfundidos (RR) de 8 ratos estudados.

\begin{tabular}{ccc}
\hline Retalhos Cutâneos & MDA (nmoles/10 mg) & N $^{\mathbf{0}}$ de retalhos \\
\hline RC & $34,50(16.0-73,0)^{\mathrm{f}}$ & 8 \\
RI & $54,35(21,8-90,0)$ & 8 \\
RR & $84,25(47,8-115,0)^{\mathrm{f}}$ & 8 \\
\hline
\end{tabular}

$$
\mathrm{RC}<\mathrm{RR}(\mathrm{p}<0,05)^{\mathrm{f}}
$$

Observa-se tendência de aumento dos níveis de MDA nos retalhos isquêmicos (RI) e reperfundidos $(\mathrm{RR})$ em relação aos retalhos controle (RC).
Após a reperfusão, os níveis de MDA aumentaram significativamente em relação aos controles (Figura 1).

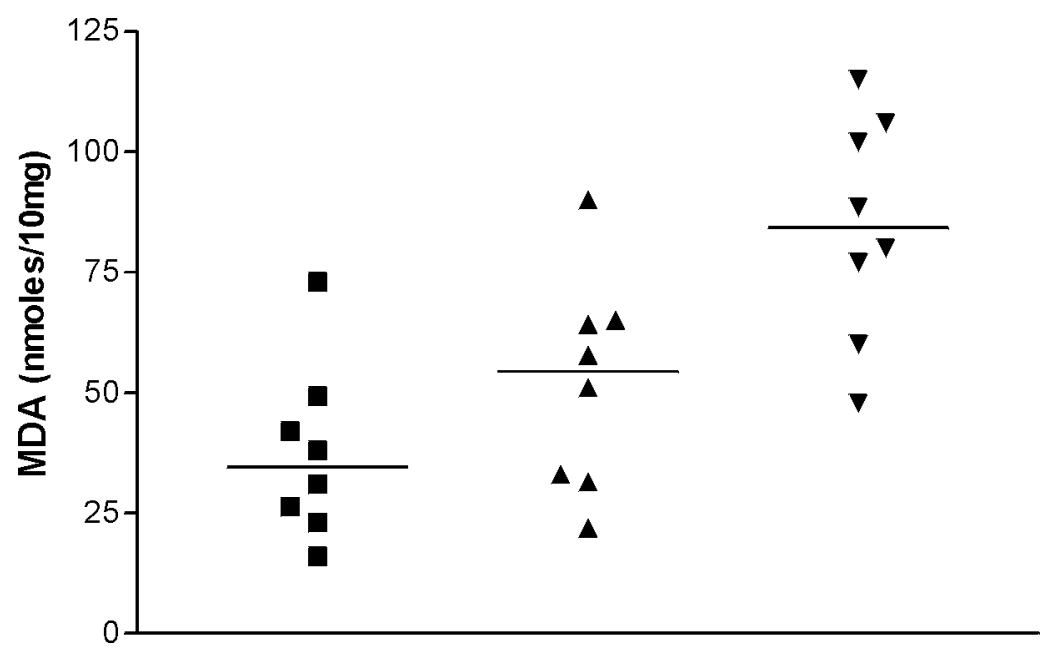

\section{Retalhos ( $n=8)$}

$$
\begin{aligned}
& R C \boldsymbol{R}=\text { retalhos controle } \\
& R I \wedge=\text { retalhos isquêmicos } \\
& R R \bullet=\text { retalhos reperfundidos } \\
& R R>R C=p<0,05 \\
& \quad=\text { Mediana }
\end{aligned}
$$

Figura 1 - Concentração tissular de malonildialdeído (MDA) em retalhos cutâneos controle (RC), isquêmicos (RI) e reperfundidos (RR) de 8 ratos estudados. 
As medianas das concentrações tissulares de XO nos retalhos controle $(\mathrm{RC})$, após $16 \mathrm{~h}$ de isquemia (RI) e 45 min de reperfusão (RR) estão expostas na tabela 2 e figura 2 .

Tabela 2 - Mediana das concentrações tissulares de xantina oxidase- XO em retalhos cutâneos controles (RC), isquêmicos (RI) e reperfundidos (RR) de 8 ratos estudados.

\begin{tabular}{ccc}
\hline Retalhos Cutâneos & XO (pmoles/10 mg) & No de retalhos $^{\text { }}$ de \\
\hline RC & $73,35(60,0-77,3)^{\mathrm{f}}$ & 8 \\
RI & $73,30(60,0-76,5)$ & 8 \\
RR & $74,20(65,0-81,1)^{\mathrm{f}}$ & 8 \\
\hline
\end{tabular}

$\mathrm{RC}<\mathrm{RR}(\mathrm{p}<0,05)^{\mathrm{f}}$

Figura.2

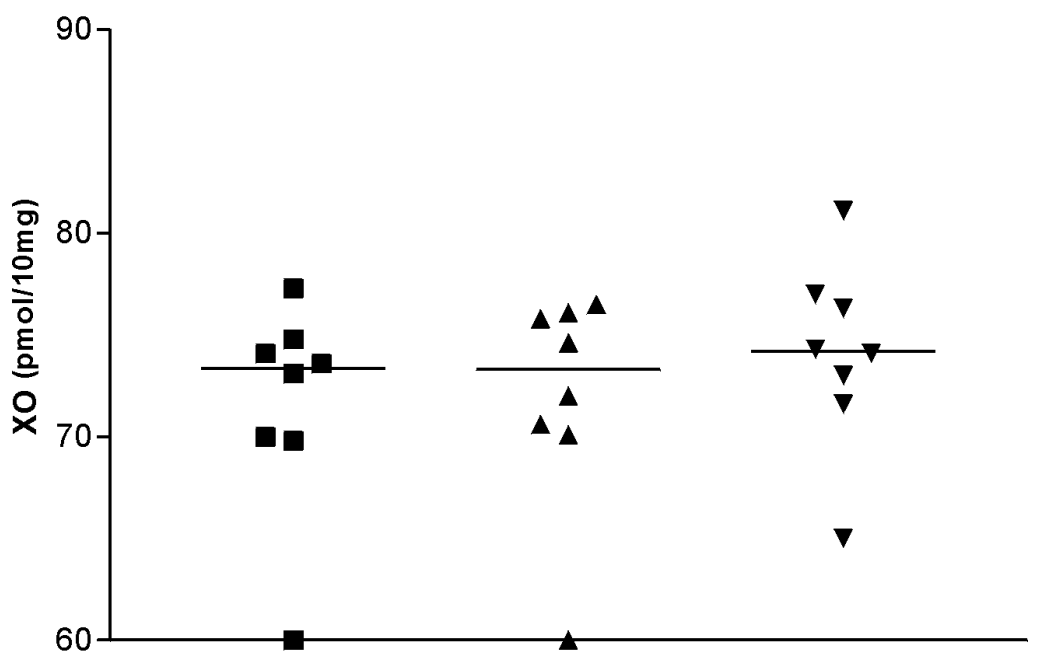

$=$ Mediana
$R R>R C=p<0,05$
$R C \square=$ retalhos controle
$R I \wedge=$ retalhos isquêmicos
$R R \nabla=$ retalhos reperfundidos

Retalhos $(n=8)$

Figura 2 - Concentração tissular de xantina oxidase $(\mathrm{XO})$ em retalhos cutâneos controle (RC), isquêmicos (RI) e reperfundidos (RR) de 8 ratos estudados.

Observou-se aumento significativo na atividade da $\mathrm{XO}$ nos retalhos reperfundidos (RR) quando comparada com a dos controles (RC). Observa-se tendência de aumento da atividade da $\mathrm{XO}$ nos retalhos isquêmicos (RI) e reperfundidos (RR) em relação aos retalhos controle (RC).

\section{DISCUSSÃO}

O retalho epigástrico de rato demonstrou ser viável no estudo da isquemia e reperfusão em tecido cutâneo.
A bilateralidade e independência do pedículo vascular torna possível a aplicação de dois retalhos no mesmo animal, sendo um deles controle e o outro submetido à isquemia/reperfusão .

O tempo de isquemia arterial de $16 \mathrm{~h}$ utilizado no presente estudo foi maior em relação a outros trabalhos, onde nestes, utilizou-se modelo de isquemia total ou apenas venosa ${ }^{16,17}$. A reperfusão de $45 \mathrm{~min}$. representa o período, onde há maior formação de $\mathrm{ERO}^{18}$.

O tempo de isquemia crítica é de $8 \mathrm{~h}$, após o qual o retalho epigástrico do rato não sobrevive, apesar da 
restauração do fluxo sangüíneo ${ }^{9,19}$. Neste estudo, optou-se pelo modelo de isquemia arterial, com oclusão temporária da artéria femoral (sem oclusão venosa) pelo tempo de 16 horas.

Como não havia necessidade de se avaliar a viabilidade da pele do retalho e o tempo de observação para a coleta de biópsias foi menor que $24 \mathrm{~h}$, optou-se por não interpor material aloplástico entre o retalho $\mathrm{e}$ seu leito. Estudos sugerem que neste período não há, sob o retalho, neovascularização, que possa interferir na interpretação dos resultados ${ }^{19}$.

O aumento de MDA, após a reperfusão nos retalhos reperfundidos, sugere que a lipoperoxidação de membranas foi mais intensa ao final deste período e houve tendência ao aumento da lipoperoxidação após a isquemia.

O MDA é o produto final da lipoperoxidação e tem sido considerado marcador de lesão tissular. Os valores encontrados no presente estudo estão de acordo com outros trabalhos publicados ${ }^{2,20,21}$.

A pele e o músculo esquelético do rato apresentam grande quantidade de xantina oxidase; assim, considerase a pele deste animal como bom modelo experimental para o estudo desta via de produção de radicais livres ${ }^{16,22}$.

A variabilidade de concentração de $\mathrm{XO}$ entre as espécies faz com que a extrapolação dos resultados em modelos animais para situações clínicas no homem seja cautelosa. Entretanto autores reportaram níveis significativos de XO em tecidos humanos, principalmente fígado e intestino delgado ${ }^{23}$.

Estudos prévios comprovaram o aumento dos níveis de XO em tecido cutâneo isquêmico e reperfundido de ratos ${ }^{2}$.

No presente estudo a atividade da XO foi maior ao final da reperfusão.

Existem evidências indicando que as ERO produzidas pelas células endoteliais submetidas à isquemia/reperfusão (via XO) e pelos neutrófilos ativados (via NADPH oxidase) têm papel importante na aderência e infiltração neutrofílica, perpetuando a reação celular em cadeia na lesão de isquemia/reperfusão ${ }^{4}$.

Conclui-se que a isquemia de $16 \mathrm{~h}$ e reperfusão de $45 \mathrm{~min}$, em retalhos epigástricos de ratos, provocam elevação de MDA e XO, caracterizando o aumento significativo da lipoperoxidação após a reperfusão.

\section{REFERÊNCIAS}

1. Manson PN, Anthenelli RM, IM M.J. The role of oxygen free radicals in ischemic tissue injury in island skin flaps. Ann Surg 1983;198(1):87-90.

2. Angel MF, Ramasastry SS, Swartz WM. The critical relationship between free radicals and degrees of ischemia: evidence for tissue intolerance of marginal perfusion. Plast Reconst Surg 1988;81(2):233-9.

78 - Acta Cirúrgica Brasileira - Vol 17 (Suplemento 3) 2002
3. Slater TF. Overview of methods used for detecting lipid peroxidation. Methods Enzymol 1984;105:283.

4. Granger DN, Kvietys PR, Perry MA. Leukocyte- endothelial cell adhesion induced by ischemia and reperfusion. Can J Physiol Pharmacol 1993;71:67-75.

5. Welbourn CRB, Goldman G, Paterson IS. Pathophysiology of ischaemia reperfusion injury : central role of the neutrophil. Br J Surg 1991;78(6):651-5..

6. Lee C, Kerrigan CL, Tellado JM. Altered neutrophil function following reperfusion of an ischemic myocutaneous flap. Plast Reconst Surg 1992;89(5):916-23.

7. Kerrigan CL, Stotland MA. Ischemia - reperfusion injury : a review. Microsurgery 1993;14:165-75.

8. Sagi A, Ferder M, Levens D, Strauch B. Improved survival of island flaps after prolonged ischemia by perfusion with superoxide dismutase. Plast Reconst Surg 1986;77(4):639-42.

9. Harashima T, Sawada Y, Watanabe S. The relationship between venous occlusion time in island flaps and flap survivals. Plast Reconst Surg 1991;44:500-5.

10. Finseth F, Cutting C. An experimental neurovascular island skin flap for the study of the delay phenomenon. Plast Reconst Surg 1978;61(3):412-9.

11. Petry JJ, Wortham KA. The anatomy of the epigastric flap in the experimental rat. Plast Reconst Surg 1984;74(3):410-3.

12. Acland R. Signs of patency in small vessel anastomosis. Surgery 1972;72(5):744-8.

13. Draper HH, Hadley M. Malonyldialdehyde determination as index of lipid peroxidation. Methods Enzymol 1990;186:42130 .

14. Im M.J, Shen WH, CJ. Effect of allopurinol on survival of hyperemic island skin flaps. Plast Reconst Surg 1984;73(2):276-78.

15. Hollander M, Wolfe DA. Non- parametric statistical methods. John Wiley \& Sons, New York, 1973.

16. Im MJ, Hoopes JE, Yoshimura Y. Xanthine: acceptor oxidoreductase activities in ischemic rat skin flaps. J Surg Res 1989;46(3):230-4.

17. Tsuzuki K, Yanai A, Tange I. The influence of congestion and ischemia on survival of an experimental vascular pedicle island flap. Plast Reconst Surg 1989;84(5):789-93.

18. Marzella L, Jesudass RR, Manson PN. Functional and structural evaluation of the vasculature of skin flaps after ischemia and reperfusion. Plast Reconst Surg 1988;81(5):742-50.

19. Sasaki GH, Pang CY. Hemodynamics and viability of acute neurovascular island skin flap in rats. Plast Reconst Surg 1980;65(2):152-8.

20. Cetinkale A, Bilgic L, Bolayirli M. Involvement of neutrophils in ischemia - reperfusion injury of inguinal island skin flaps in rats. Plast Reconst Surg 1998;102(1):153-60.

21. Cengiz C, Köse AA, Aral E. Protective effect of fucoidin (a neutrophil rolling inhibitor) on ischemia reperfusion injury: experimental study in rat epigastric island flaps. Ann Plast Surg 2001;47(5):540-6.

22. Parks DA, Granger DN. Xanthine oxidase: biochemistry, distribuition and physiology. Acta Physiol Scand. Suppl 1986;548:87-99.

23. Krenitsky TA, Tuttle JV, Cattan EL A comparision of the distribution and electron acceptor specificities of xanthine oxidase and aldehyde oxidase. Comp Biochem Physiol 1974;49B:687-703.

\section{Endereço para correspondência}

\section{Carlos Eli Picicnato}

Departamento de Cirurgia e Anatomia

Faculdade de Medicina de Ribeirão Preto

14049-900 - Ribeirão Preto - SP

e-mail cepiccin@fmrp.usp.br 